\title{
FLUID-SOLID COUPLED SIMULATION AND EXPERIMENTAL VALIDATION OF F FLUID FLOW IN LIQUID DISTRIBUTOR
}

\author{
SUI Xiu-hua ${ }^{1}$, Wang Li-feng ${ }^{2}$, Wang Shao-bo ${ }^{3}$ \\ ${ }^{1,2,3}$ College of Mechanical and Electronic Engineering, Shandong University of Science and Technology, \\ Qingdao,China, suixh@126.com,wanglifeng7011@163.com,wshb1987@126.com
}

\begin{abstract}
Liquid distributors which on the top the wet curtain are widely used in industry field especially in the packed tower and solution dehumidifier. Consequently, liquid distributors play a major role. Many scholars devote to design and analyze the performance of liquid distributor in order to improve distribute liquid capacity of the liquid distributor. The velocity or the flow of the outlet is very important for the performance of the liquid distributor which directly affects the performance of the solution dehumidifier, however, the velocity is difficult to quantify unless the flow field is known. Therefore outflow through the outlet of liquid distributors has been simulated using computational fluid dynamics (CFD) method.
\end{abstract}

As first part of the project a new kind of liquid distributor which is used mine solution dehumidifier is proposed based on the related theory.

In a second part of the project, through the Fluid-solid coupled simulation method, the velocity of the liquid distributor outlet and the stress of the orifice plate were analyzed based on the Fluid-solid coupled theory. The velocity and the stress were obtained which indicated that orifice plate liquid distributor had the well distribute liquid capacity. These results were validated by experimental results.

In a third part of the project, the experiment was carried out to analyze the capacity of the orifice plate liquid distributor.

In this paper, the performance of the liquid distributor of the mine solution dehumidifier was analyzed by the simulation and experimental methods, which provide a new idea and method for the design and analysis of distributor.

Index Terms: distribute liquid capacity; liquid distributor; Fluid-solid coupled simulation; experiment; flow;

\section{INTRODUCTION}

The liquid distributor which is located in the top of the wet curtain is one of the key components of the solution dehumidifier, its function is provide larger contact area for gas-liquid contact, so that the hygroscopic properties of the solution could fully exploited, However, when the design of the liquid distributor is unreasonable, it will result in The uniform distribution is not very well as well as the wetting degree of wet curtain is inconsistent, solution dehumidifier performance cannot be fully exploited. Therefore, the uniform distribution of liquid above wet curtain packing is very important for the solution dehumidifier performance.

There are many scholars devote to design and analyze the performance of liquid distributor. Gao[1] reviewed the distribution performance of the LPT-1 liquid distribution in the test device, learned that LPT-type liquid distributor can increase the operating elasticity of the vacuum distillation tower, reduce fuel consumption, lower vacuum distillation tower pressure drop and improve extraction rate. Song [2] researched and designed a new type liquid distributor-fractal liquid distributor. Through the experiment learned that it can not only ensure geometric uniformity of the liquid spray point distribution, but also guarantee the flow rate uniformity of spray point. Luo [3] gave the design method of row tube type liquid distributor based on the simplified fixed momentum equations. Duan[4] simulated internal flow field of the impact spray thrower by using Fluent software and obtained the regularity of the factors that influence efficiency of spray. B.Y. Guo [5] pointed out that the inlet velocity is the key factor which effects liquid export velocity uniform. Almendros [6] presented a new kind distributor called the rotating liquid distributor as well as analyzed its performance. Based on their results, the performance of the rotating liquid distributor is related to the fluid velocity, flow, and rotational speed liquid distributor. Lothar[7] proposed the concept of wetting coefficient, utilizing wetting coefficient to characterize liquid distributor distribute capacity. Dong [8] used the so-called discrete particle simulation to take account of particle-particle and particle-wall interactions and to track 
each particle. Their work has been able to provide de-tailed particle-scale information for the particle flow.

The main work of this paper is putting forward a new kind liquid distributor which is used in the solution dehumidifier, The fluid-structure coupled simulation has been carried based on the fluid-structure coupled theory simulate row tube type liquid distributor internal flow field .Through the simulation, the velocity distribution of the outlet and the stress of the liquid distributor are obtained. At last verify the feasibility of orifice plate liquid distributor through the experiment and simulation.

\section{FLUID-SOLID COUPLED SIMULATION}

\subsection{The Objects of Simulation}

An ideal liquid distributor should distribute the liquid uniform, own large free area, have wide operating flexibility, the orifice plate liquid distributor is designed which could use in the solution dehumidifier according to the above principles and relevant design theory.

The orifice plate liquid distributor belongs to the gravity-type liquid distributor, liquid depend on the action of gravity flows from the orifice.

The flow of the outlet is expressed as:

$$
q=\frac{\pi}{4} d_{0} c \sqrt{2 g h}
$$

Solution flow conservation equation:

$$
Q=n q
$$

The number $\mathrm{n}$ of the orifice can be drawn from (1-1) and (12):

$$
n=\frac{Q}{\frac{\pi}{4} d_{0} c \sqrt{2 g h}}=\frac{4 Q}{\pi d_{0} c \sqrt{2 g h}}
$$

Because the real liquid distributor is to complex when simulate in the CFX, so simplified distributor model is put forward in order to evaluate the performance of the orifice plate liquid distributor by simulation and experiment easily.

The size of model is: $150 \mathrm{~mm} \times 100 \mathrm{~mm} \times 20 \mathrm{~m}$.

The total flow: $2.3 m^{3} / h$.

According to the design of the liquid distributor, take the diameter of the orifice is $5 \mathrm{~mm}$.
According to the formula (1-3), numbers of orifices are 104. Open the orifices at the bottom of the orifice plate liquid distributor and the diameter of the orifice is $5 \mathrm{~mm}$.

The orifice plate liquid distributor model is shown in Fig 1. The liquid model is shown in Fig 2.

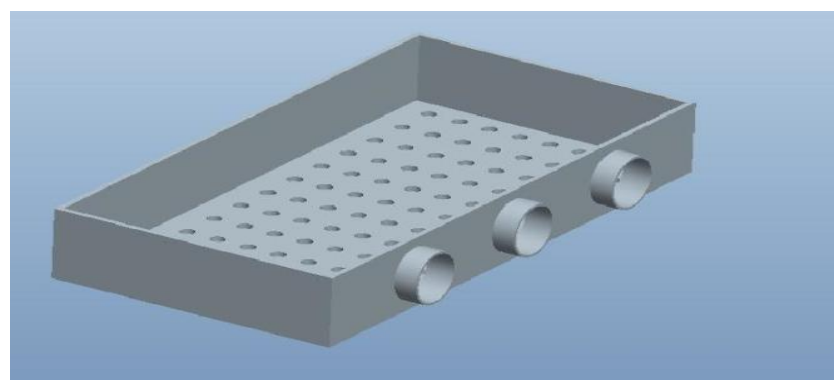

Fig1 the orifice plate liquid distributor

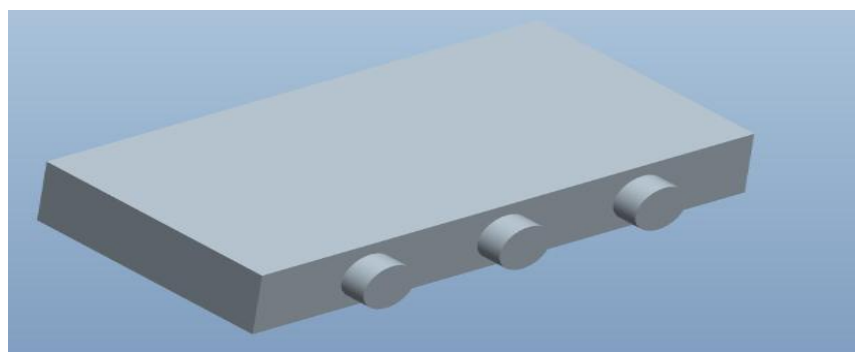

Fig 2 the liquid model

\subsection{Fluid-Solid Coupled Simulate Theory}

Solids will produce deformation or movement under the fluid dynamic loads; however the deformation or movement of the solids in turn will affect the flow field which would change the distribution of the velocity and size of the fluid load.

Stress distribution of the orifice plate liquid distributor and flow flied distribution of the liquid are investigated using the Fluid-solid coupled method.

No matter the fluid or the solid, they all comply with the appropriate physical principles and laws.

\section{(1) Fluid control equation}

1) Flow conservation equation:

$$
\frac{\partial \rho_{f}}{\partial t}+\nabla \cdot\left(\rho_{f} v\right)=0
$$

2) Fluid momentum conservation equation:

$$
\frac{\partial \rho_{f} v}{\partial t}+\nabla \cdot\left(\rho_{f} v v-\tau_{f}\right)=f_{f}
$$


3) Fluid energy conservation equation:

$$
\begin{aligned}
& \frac{\partial\left(\rho h_{h o t)}\right.}{\partial t}-\frac{\partial p}{\partial t}+\nabla \cdot\left(\rho_{f} v h_{h o t}\right) \\
& =\nabla \cdot(\lambda \nabla T)+\nabla \cdot(v \cdot \tau)+v \cdot \rho f_{f}+S_{E}
\end{aligned}
$$

\section{(2) Solids control equation}

1) The conservation equation of solid part can export form Newton's second law :

$$
\rho_{s} \overline{d_{s}}=\nabla \cdot \sigma_{s}+f_{s}
$$

2) $f_{T}$, Solid deformation caused by temperature:

$$
f_{T}=\alpha_{T} \cdot \nabla T
$$

3) Liquid -structure coupled equation

Fluid-structure interaction also follow the conservation equations, the fluid and solid stress $\tau$, conservation displacement $\mathrm{d}$, heat flow $\mathrm{q}$, temperature $\mathrm{T}$ which in the fluidstructure interaction surface also meet the conservation equations.

$$
\begin{gathered}
\tau_{f} \cdot n_{f}=\tau_{s} \cdot n_{s} \\
d_{f}=d_{s} \\
q_{f}=q_{s} \\
T_{f}=T_{s}
\end{gathered}
$$

\subsection{Parameter Settings}

Set boundary conditions and parameters for the established model.

First, define the fluid domain parameters.1) Use steady-state simulation, the fluid is water which temperature is $13^{\circ} \mathrm{C}$. 2)The boundary conditions: the definition of inlet velocity boundary, $2 \mathrm{~m} / \mathrm{s}$, outlet conditions uses pressure boundary, the relative pressure $0 \mathrm{~atm}$. 3)Taking into account the heat transfer, open the heat transfer model: the Total Thermal.

Second, define the liquid distributor parameters. 1)Define the liquid distributor material PVC; 2)Define the fix surface, because the lateral surfaces close to the inner wall of the solution dehumidifier, so lateral surface of the distributor cannot move or deformation, thus define fix type of lateral surface is full Constraint; 3) The forces of rest wall are given by fluid. Import the calculated fluid load to liquid distributor through imported load command. The import fluid pressure and import fluid temperature are shown as Fig3 and Fig; 4), Utilize the multi-field solver MFS to solve.

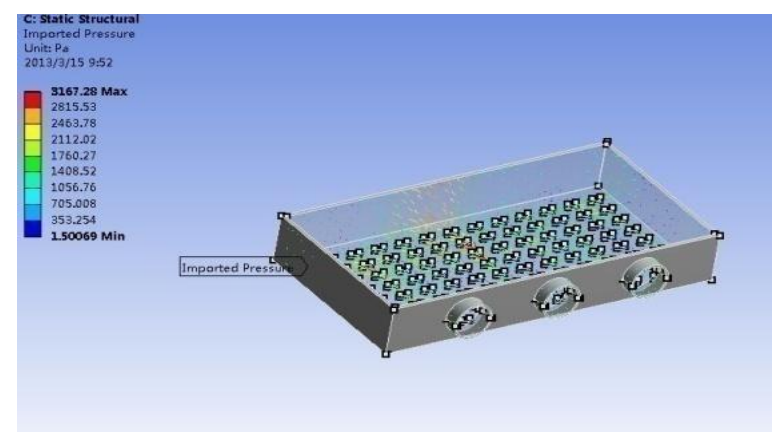

Fig3: Import fluid pressure

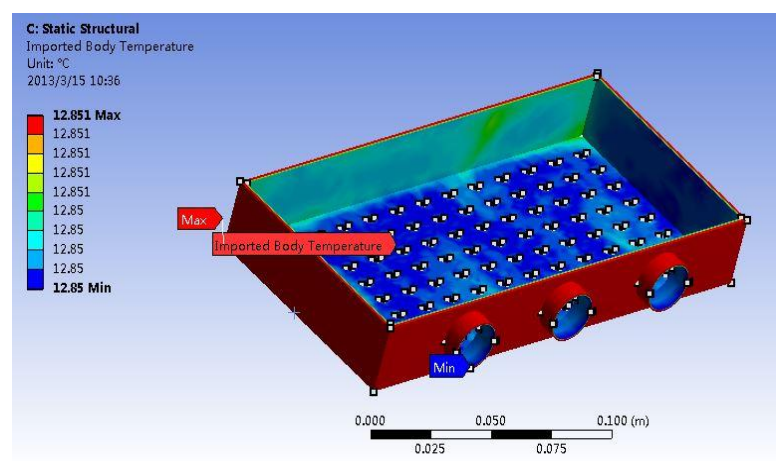

Fig4: Import fluid Temperature

\subsection{Simulation Results}

Fluid velocity counter plot as well as stress and displacement of the liquid distributor counter plot are obtained by coupling simulation.

The velocity distribution of the outlet indicates distribute liquid capacity of the orifice plate liquid distributor.

The velocity counter plots of the different cross-area are shown as Fig5 and Fig6.

\section{(1) Velocity distribution}

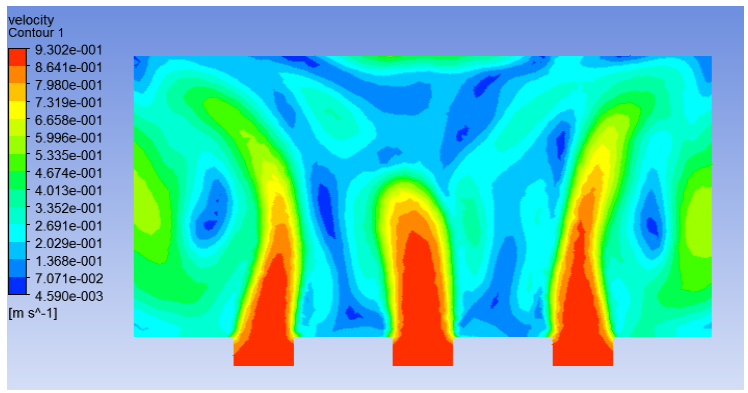

Fig5 velocity counter $\operatorname{plot}(\mathrm{z}=-10 \mathrm{~mm})$ 


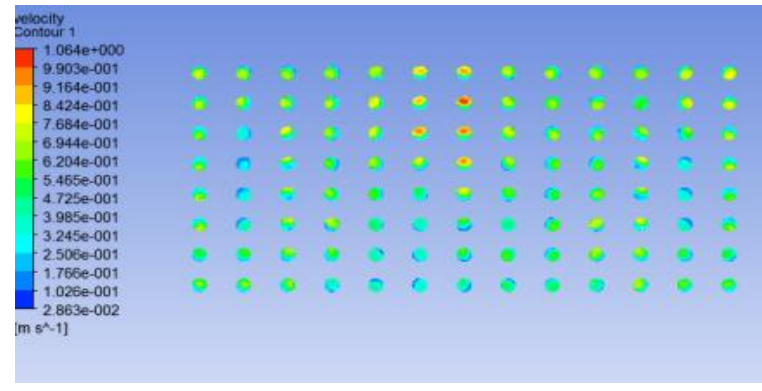

Fig6 velocity counter $\operatorname{plot}(\mathrm{z}=-20 \mathrm{~mm})$

Fig5 shows that a certain thickness of the layer of water forms in the inside of the liquid distributor after the liquid flow into the liquid distributor. The water which has a certain momentum and energy emitted out at a certain speed. Water outflow from the lateral inlet pipe formed reflux near the inner wall of the liquid distributor and water outflow from the middle inlet pipe appeared Jet flow morphology. Jet flow and reflux influence each other which make the liquid distribution are very complex in the liquid distributor. Thus, it was difficult to describe the fluid field in the math method. The Fig 6 shows that the velocity of outlet generally consistent, the velocity of the outlet no obvious jumps or is very small. However, the velocity of outlets which locate at the second row on the left and the second row on the right side speed is lower, about $0.5-0.6 \mathrm{~m} / \mathrm{s}$. The outlets that the speed is small are consistent with reflux region. The velocity of the outlets which far from the inlet pipe is larger than the velocity of the outlets which near the inlet pipe. The Average speed of liquid distributor is $0.8 \mathrm{~m} / \mathrm{s}$.

\section{2 ) Stress and displacement counter plot of the liquid distributor}

Stress indicates the forces size that Liquid fluid applied to liquid distributor.

Liquid distributor deformation will change the orifice area which will affect fluid uniformity.

The displacement and stress counter plot are shown in Fig 7 and Fig 8.

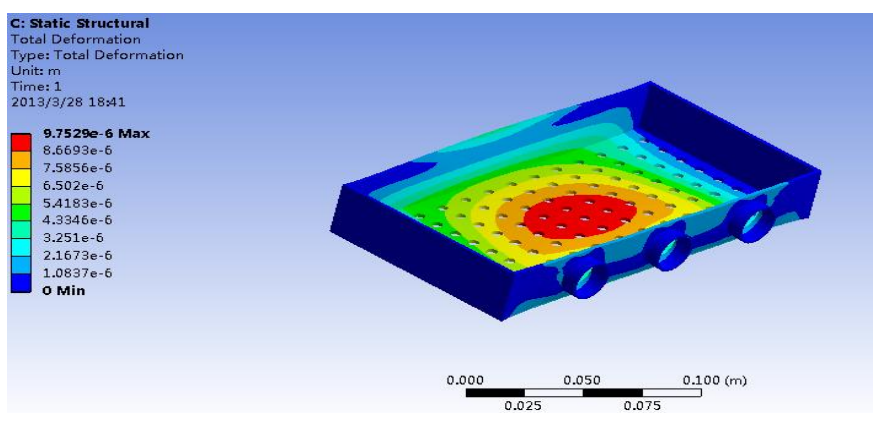

Fig7 displacement counter plot

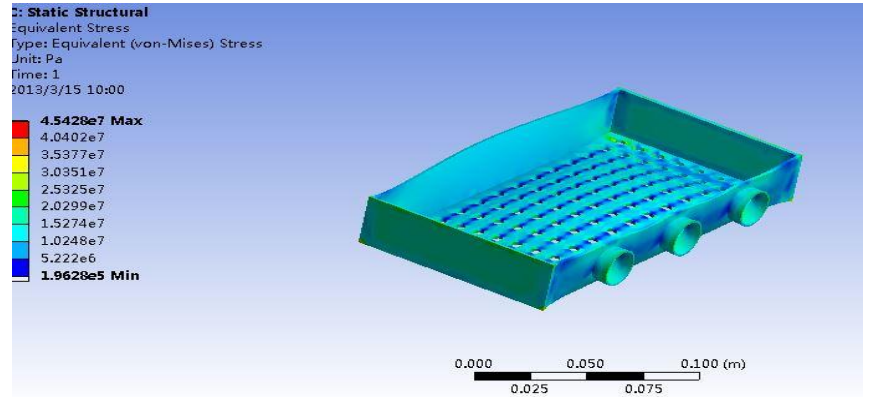

Fig8 stress counter plot

The Fig 7 shows that the deformation of the liquid distributor mainly concentrated in two parts: the bottom surface of the liquid distributor and the front wall of the liquid distributor. Although the deformation of the liquid distributor will change the outlet diameter, the maximum deformation is only $8.66 \mathrm{e}-$ $3 \mathrm{~mm}$, simulation results indicates that the deformation will not affect the distribution uniform. The Fig8 shows overall stress distribution liquid distributor is uniform which does not appear the phenomenon stress concentration. Stress distribution of bottom surface is uniform, lateral stress is larger than the stress of the bottom. The value of the lateral stress is $2.2 \mathrm{Mpa}$ which is smaller than the PVC material allowable stress $(25 \mathrm{Mpa})$. So the material that we select is no problem. Although the stress of the bottom surface is smaller, the deformation is larger, because the liquid temperature is low, under the combined effect of the stress and the character of the material that the material expands with heat and contracts with cold, thus the deformation of the bottom is more intense. The left and right surfaces are constrained, so its deformation is zero. The reason that causes the front and back walls deform include two aspects: one is the liquid stress, on the other hand, impacted by the character of the material that the material expands with heat and contracts with cold.

\section{EXPERIMENTS}

Experiment was carried out to validate the performances of physical model; Fig 9 shows experiment devices of orifice plate liquid distributor. The volume flow rate of pump is $2.3 m^{3} / h$.

The water was sent to the liquid distributor by the pump, the liquid that flowed through the liquid distributor returned to the water tank.

Utilizing the measuring cylinder to catch the flow of the liquid distributor orifice, studying the distributed performance of the orifice plate liquid based on the amount of water in the graduated cylinder distributor. According to the principle of uniform, the points are selected, select six points $n$ the length direction, select three points along the width direction. The experimental device is shown in the Fig10, experimental data, is shown in Table 1: 


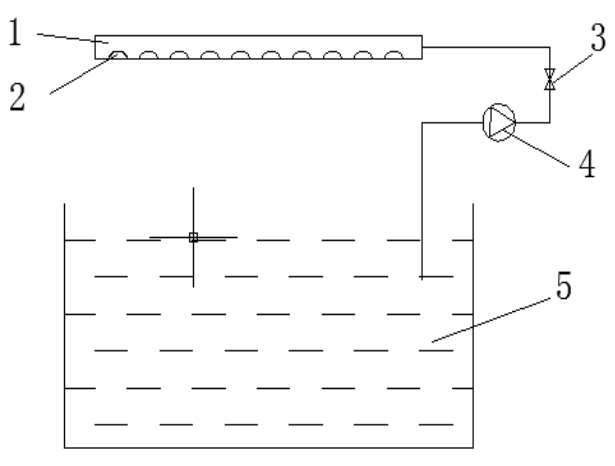

Fig 9 the experimental device

1- Orifice plate liquid distributor, 2-orifice, 3-valve, 4-pump, 5- water tank

\begin{tabular}{|lllllllllllll|}
\hline 0 & 0 & 0 & 0 & 0 & 0 & 0 & 0 & 0 & 0 & 0 & 0 & 0 \\
0 & 1 & 0 & 2 & 0 & 3 & 0 & 4 & 0 & 0 & 0 & 0 & 0 \\
0 & 0 & 0 & 0 & 0 & 0 & 0 & 0 & 0 & 0 & 0 & 0 & 0 \\
0 & 0 & 0 & 8 & 0 & 9 & 0 & 10 & 0 & 11 & 0 & 12 & 0 \\
0 & 0 & 0 & 0 & 0 & 0 & 0 & 0 & 0 & 0 & 0 & 0 & 0 \\
0 & 0 & 0 & 0 & 0 & 0 & 0 & 0 & 0 & 0 & 0 & 0 & 0 \\
0 & $1 B$ & 0 & 14 & 0 & 15 & 0 & 16 & 0 & 17 & 0 & 18 & 0 \\
0 & 0 & 0 & 0 & 0 & 0 & 0 & 0 & 0 & 0 & 0 & 0 & 0 \\
\hline
\end{tabular}

Fig 10 measurement places

Table1 data of measurement, $\mathrm{ml} / \mathrm{s}$

\begin{tabular}{|c|c|c|c|c|c|c|}
\hline number & 1 & 2 & 3 & 4 & 5 & 6 \\
\hline $\begin{array}{c}\text { Volume } \\
\text { flow }\end{array}$ & 6.4 & 6.5 & 6.8 & 6.7 & 6.5 & 6.4 \\
\hline number & 7 & 8 & 9 & 10 & 11 & 12 \\
\hline $\begin{array}{c}\text { Volume } \\
\text { flow }\end{array}$ & 5.8 & 5.9 & 6 & 6 & 6.2 & 5.7 \\
\hline $\begin{array}{c}\text { number } \\
\text { nolume } \\
\text { flow }\end{array}$ & 5.8 & 5.6 & 5.9 & 5.8 & 5.7 & 5.8 \\
\hline
\end{tabular}

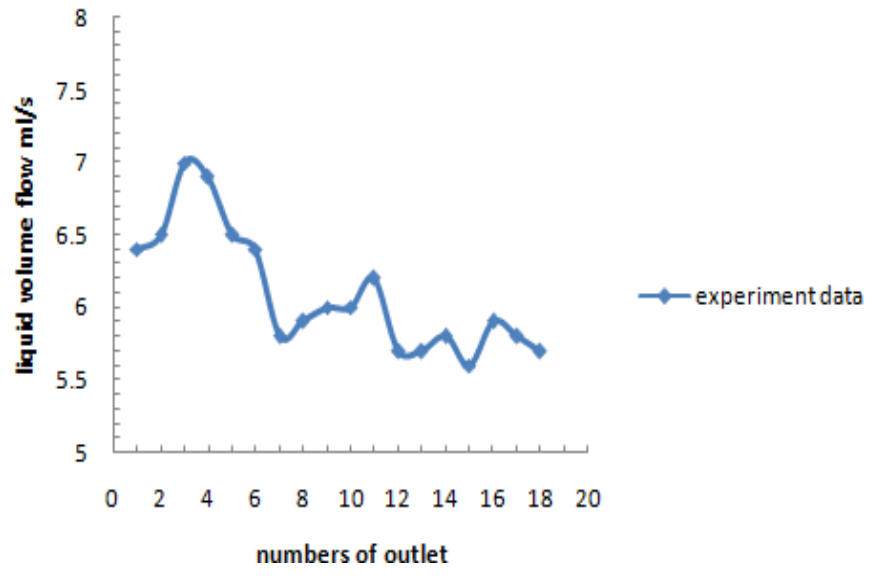

Fig 11 volume flow

The Fig 10 shows that the velocity of the back side orifice is larger than velocity of the front side orifice. The velocity of the middle points are large than both sides.

According to the uniformity coefficient formula:

$$
M_{f}=\sqrt{\frac{1}{N} \sum_{i=1}^{N}\left(\frac{Q_{i}-\overline{Q_{i}}}{\bar{Q}_{i}}\right)^{2}}
$$

Uniformity coefficient of the orifice plate liquid distributor can be calculated, $\mathrm{Mf}=0.03$

The Mf value shows that the orifice plate liquid distributor could distribute the liquid uniform.

\section{COMPARE OF SIMULATED VOLUME FLOW THROUGH THE OUTLET ORIFICES AND EXPERIMENTAL DATA}

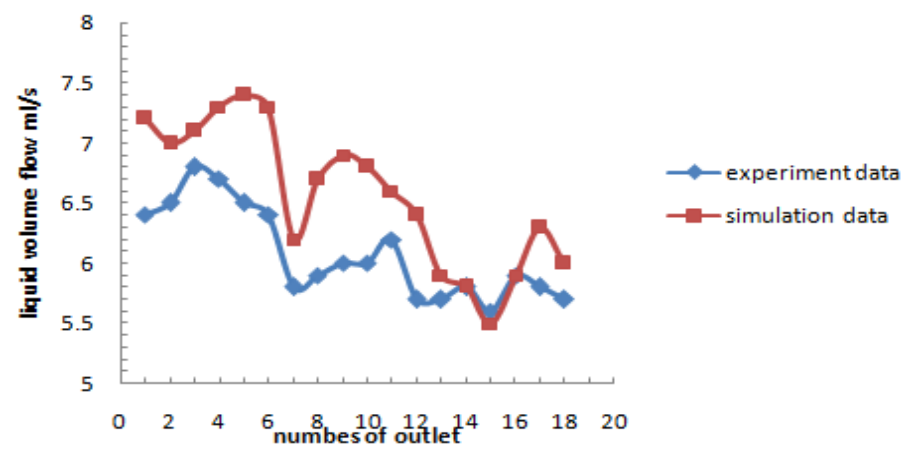

Fig 12 Compare of simulated volume flow through the outlet orifices and experimental data 
The Fig12 shows that the simulation data are large the experimental data.

The common: The simulation data curve and experimental data curve have the same variation trend. The velocity of the back side orifice is larger than velocity of the front side orifice. The velocity of intermediate points are large, the velocity of both sides points are small.

The difference: The simulation results value is greater than the experimental results, the average value of the experimental results is $6.1 \mathrm{ml} / \mathrm{s}$, however the average value of simulation results is $6.5 \mathrm{ml} / \mathrm{s}$. Simulation results variation trend is more acute.

\section{CONCLUSIONS}

The fluid-structure coupled simulation has been carried based on the fluid-structure coupled theory, the results of the simulation shows that the stress of the orifice plate liquid distributor is smaller the PVC material allowable stress which indicates that the distributor make of the PVC is practicable, through Compare of simulated volume flow through the outlet orifices and experimental data, we can know that the simulation results reflect the actual working condition of the distributor. The simulation data and experimental data illustrate that the orifice plate liquid distributor could distribute the liquid uniformity.

\section{ACKNOWLEDGEMENTS}

We are very thankful to The Natural Science Foundation of Shandong Province (Grant No.ZR2012EEM004) for sponsoring to publish this paper.

\section{NOMENCLATURE}

- $\mathrm{q}$, flow, $m^{3} / s$

- $d_{0}$ diameter of orifice, $m$

- $h$, Liquid level, $m$;

- $g$ gravity, $m / s^{2}$

- $c$, flow coefficient of the orifice, $c=0.61-0.63$

- $Q$, Total flow of the liquid, $\mathrm{m}^{3} / \mathrm{s}$;

- $n$,the number of orifice;

- $t$, time, sec;

- $f_{f}$, volume vector;

- $\rho_{f}$, solution density, $\mathrm{kg} / \mathrm{m}^{3}$;

- $\quad v$, liquid velocity, $m / s$

- $\tau_{f}$, Shea stress tensor, $\mathrm{Pa}$

- $S_{E}$ energy source term

- $\lambda$, thermal conductivity;
- $\quad p$, Liquid pressure. $\mathrm{Pa}$

- $\rho_{s}$, liquid distributor density, $\mathrm{kg} / \mathrm{m}^{3}$

- $S_{s}$, cauchy stress tensor;

- $f_{s}$, volume force vector;

- $\bar{d}_{s}$, Solid domain local acceleration, $\mathrm{m} / \mathrm{s}^{2}$

- $\mathrm{T}$, the temperature $\mathrm{K}$.

- $\alpha_{T}$, coefficient of thermal expansion

- $q_{f}, q_{s}$ heat flow $w / s$

- $\tau_{f}, \tau_{s}$ the fluid and solid stress $\mathrm{Pa}$

\section{Subscripts}

- $\quad$ f, fluid

- s, solid

\section{REFERENCES:}

[1] Gao Y F, distribution performance industrial applications of LPT-1 liquid distributor, Petroleum refining and chemical, 2011(5), 81-84.

[2] Song H Y. A New High-Efficient Fractal Liquid Distributor, Journal of petrochemical universities, 2004(1), 3740.

[3] Luo C X, Design of Calandria Liquid Distributor in Packed Columns, Journal of Changchun University of Science and Technology （Natural Science Edition, 2011(3),122-124.

[4] Duan W G, Analysis of Liquid Distributor Internal Flow Based on FLUENT, Modern manufacturing technology and equipment, 2009 (2), 17-18.

[6]B.Y. Guo, K.J. Dong, K.W. Chu , A.B. Yu, A. Vince, I. Brake, A numerical model for the liquid flow in a sputnik coal distributor, Minerals Engineering 2009 (22) 78-87

[7] Lothar Spiegel, A new method to assess liquid distributor quality, Chemical Engineering and Processing, 2006 (45) 1011-1017.

[8]R.Rautenbach, B.Arzt. Large scale diesel drive vapor compression units, Desalination, 1981(4):415-420..

[9] Dong, K.J, Guo, B.Y, Chu, K.W, Yu, A.B, Brake, I. Simulation of liquid-solid flow in a coal distributor. Discrete Element Methods (DEM)'07, August 27-29, 2007.

\section{BIOGRAPHIES:}

Sui Xiuhua, associate professor, research major: mechanical and electronic engineering, email: suixh@126.com

Wang Lifeng, master degree student, email: wanglifeng7011@163.com

Wang Shaobo, master degree student, email: wshb1987@126.com 